\title{
Monoclonal antibodies specific to heat-treated porcine blood
}

\begin{abstract}
BACKGROUND: Porcine blood is potentially being utilized in food as a binder, gelling agent, emulsifier or colorant. However, for certain communities, the usage of animal blood in food is strictly prohibited owing to religious concerns and health reasons. This study reports the development of monoclonal antibodies (MAbs) against heat-treated soluble proteins (HSPs) of autoclaved porcine blood; characterization of MAbs against blood, non-blood and plasma from different animal species using qualitative indirect non-competitive enzymelinked immunosorbent assay (ELISA); and immunoblotting of antigenic components in HSPs of porcine blood.
\end{abstract}

RESULTS: Fifteen MAbs are specific to heat-treated and raw porcine blood and not crossreacted with other animal blood and non-blood proteins (meat and non-meat). Twelve MAbs are specific to porcine plasma, while three MAbs specific to porcine plasma are cross-reacted with chicken plasma. Immunoblotting revealed antigenic protein bands ( 60, 85ї 100 and $\sim 250[\mathrm{WH}$ ) in porcine blood and plasma recognized by the MAbs.

CONCLUSION: Selection of MAbs that recognized 60[सWa HSPs of porcine blood and plasma as novel monoclonal antibodies would be useful for detection of porcine plasma in processed food using the immunoassay method.

Keyword: Porcine blood; Monoclonal antibody; Heat-treated soluble blood proteins; Immunoassay 